\title{
Urgences
}

\section{Heiner Müller, Hamlet-machine précédé de Mauser et autres textes, traduction de l'allemand par Jean Jourdheuil et Heinz Schwartzinger, Paris, Minuit, 1979, 88 p.}

\section{Renald Bérubé}

Numéro 17-18, octobre 1987

L'esprit des lieux

URI : https://id.erudit.org/iderudit/025436ar

DOI : https://doi.org/10.7202/025436ar

Aller au sommaire du numéro

Éditeur(s)

Urgences

ISSN

0226-9554 (imprimé)

1927-3924 (numérique)

Découvrir la revue

Citer ce compte rendu

Bérubé, R. (1987). Compte rendu de [Heiner Müller, Hamlet-machine précédé de Mauser et autres textes, traduction de l'allemand par Jean Jourdheuil et Heinz Schwartzinger, Paris, Minuit, 1979, 88 p.] Urgences, (17-18), 208-209.

https://doi.org/10.7202/025436ar d'utilisation que vous pouvez consulter en ligne.

https://apropos.erudit.org/fr/usagers/politique-dutilisation/ 
maux, scènes, silences, jeux, tous issus d'un univers de substances concret et fluide, fait pour être entrevu et ressenti: Bleu de lessive et coulis de framboises, [...] le monde est en saveurs,(p. 39) rendant le monde soudain proche de la parole.

«Certains plaisirs se croquent comme un bonbon» écrit France Huser. Coqs à deux têtes est de ceux-là, il faut savoir savourer (ma mère/de phrases s'eniure de voyelles de lilas,(p. 34) en écoutant les mots qui résonnent,(p. 21 ) les mots qui se font la nique ou la cour(se): une histoire d'amour (des mots) à l'envers du temps, un livre inactuel et sur _ctuel donc.

1. Paul Chanel Malenfant: «De tels effets de langue», La nouvelle barre du jour, no 175, avril 1986, p.15.

2. Eléments les plus apparents à première lecture. II faudrait aussi mentionner, par exemple, Le tambour de Günther Grass.

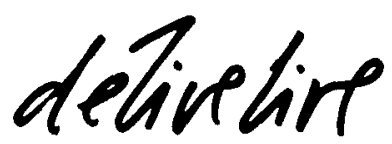

\section{Heiner Müller: Hamlet-machine précédé de Mauser et autres textes, (traduction de l'allemand par Jean Jour- dheuil et Heinz Schwartzinger), Paris, Minuit, 1979, 88 p.}

Tout petit livre, donc, 88 pages, format folio (à peu près). La table des matières n'en contient pas moins neuf titres: *textes" (selon la pagecouverture) ou «pièces* (selon la page de titre) qui pratiquent divers genres et dont les dates de composition vont de la fin des années 1950 à un moment indéterminé de celles-ci en passant par les années 1960 et 1970 . La table des matières se présente (donc) ainsi, à quoi s'ajoutent ici, pour chacun des textes, les indications génériques et chronologiques données en page 4 et je souligne les titres ainsi que le fait ladite page: le Père (nouvelle, 1958); Deux Lettres (poème, 1956); Avis de décès (nouvelle, 1975); Horace (pièce,
1968); Mauser (pièce, 1970); Adieu à la pièce didactique (lettre(?), 1977); Hamlet-machine (pièce, 1977); Autoportrait deux heures du matin le 20 août 1959 (poème); Projection 1975 (poème, aécrit dans les années $50 x$, p. 85). Questions-remarques (discussion soliloquée) portant sur deux détails, les détails ayant souvent l'astuce et l'importance que l'on sait: faut-il bien souligner tous ces titres? Ne vaudrait-il pas mieux, dans certains cas (lesquels?), les placer entre guillemets? Je veux dire que les guillemets convient l'idée de recueil, connotent l'idée de partie(s) d'un ensemble, le souligné ayant plutôt tendance à conférer de l'autonomie. Je sais: il est possible de conserver son 
autonomie tout en habitant un ensemble, sinon même en le construisant, et Lumière d'août n'en est pas moins un moment du parcours faulknérien. Bien sûr. Mais ce livre de Müller ayant l'architecture qui est la sienne, il vaut la peine de (il nous oblige à) souligner ce premier détail. Par ailleurs, la page 4 affirme que les deux derniers textes ont été écrits $\alpha$ au début des années $50 \nsim$; il semble pourtant que le titre de l'avantdernier et l'indication chronologique accompagnant le dernier ne donnent pas le même avis. Alors? (La discussion, portant sur des détails, peut s'arrêter ici.)

Livre bref, cela a été dit, qui sait jouer du parcours chronologique des dates de composition de ses textes et de la pratique de genres divers; qui, multipliant en plus les titres qui le composent, instaure (donc) la rupture en se multipliant. C'est-à-dire/mais aussi: livre dense, réfléchi, construit, qui oblige à une lecture lente, attentive, qui sache revenir sur elle-même et se remettre en cause, aux aguets et minutieuse. Vous lisez, écrit en italiques, le début du premier texte: "Un père mort eût été peut-être/Un meilleur père. Le mieux/C'est le père mort-né.» (p. 7), puis, votre lecture s'achevant, la fin du dernier texte: $\star$ Je me rappelle ma première tentative d'écrire une pièce. Le texte s'est perdu dans les troubles de l'après-guerre. Ça commençait par le héros (jeune) devant le miroir qui cherchait à deviner quel chemin les vers emprunteraient dans sa chair. À la fin, il était dans la cave et découpait son père. Au siècle d'Oreste et d'Électre, qui monte, Oedipe sera une comédie» ( $p$. 85); le trajet incipit-explicit, raccourci exemplaire ici, réaffirme à sa manière les constatations du parcours lectoral entier, c'est-à-dire que ce petit livre, de ruptures en courts-circuits, tisse sa cohérence et sa continuité, son organisation. Le paradoxe comme moyen de réflexion. Neuf textes qui sont comme autant de fragments autonomes, d'essais qui prennent la forme d'autres genres; petit livre qui, pratiquant comme avec allégresse ou par obligation l'intertextualité et l'hypertextualité, sait d'un texte à l'autre s'interroger et se répondre ou vice versa, toujours aux frontières du silence. Müller, à la fois dans le sillage de Brecht et de Beckett.

Hamlet-machine: treize pages à peine, dont la troupe Carbone 14 de Montréal, sous la direction de Gilles Maheu, fera, au printemps de 1987, un admirable spectacle de deux heures environ. L'interprète d'Hamlet, chez Müller, a lu, vu et interrogé Hamlet de l'on sait qui, «notre maître à tous écrit $\mathrm{Hu}$ bert Aquin dans Point de fuite (Montréal), CLF, 1971, p. 76). Hamlet, grosse machine théâtrale et machine à produire du texte, dont Hamlet-machine. Hamlet, machine à écrire et à réécrire, Hamlet et la question du ou des pères, biologique(s) et politique(s). Hamletmachine qui reprend des passages de Deux lettres et dont l'Ophélie reprend et refuse le destin de la femme suicidée de Avis de décès pour devenir Electre ( $p$. 80). Le Hamlet de Müller: être (p. 79) ou ne pas être (Mauser, p. 61) une machine? Et laquelle, et donc au service de qui?

Traducteur et metteur en scène de Müller (Allemand de l'est, né en 1929), Jean Jourdheuil écrit: *Or donc, j'éprouve de l'étonnement lorsque je lis les textes de Heiner Müller, non seulement parce que ces textes me montreraient la «face cachée de la lune» (selon l'expression d'un critique amateur de littérature est-allemande, Claude Prévost si ma mémoire ne me trahit pas), mais aussi parce que l'écriture de Müller, aussi dense et brutale soit-elle, me semble être une lecture spectatrice (...). Et j'ai l'impression que c'est parce qu'elle est spectatrice que l'écriture de Müller est si provocante. Et dans ses ouvrages plus récents, Gundling Hamlet-machine, la Mission, Müller écrivain m'apparaît être devenu spectateur de Müller écrivant; comment dans ces conditions ne serait-il pas ironique à l'endroit de ses propres imprécations jusqu'à les rendre triviales et comiques. ( Passages*, dans Théâtre/Public, nos $46-47$, juilletoctobre 1982, p. 98 ).

Renald Bérubé 